\title{
Topological entanglement entropy of a Bose-Hubbard spin liquid
}

\author{
Sergei V. Isakov' ${ }^{1}$ Matthew B. Hastings ${ }^{2,3}$ and Roger G. Melko ${ }^{4 \star}$
}

The Landau paradigm of classifying phases by broken symmetries was shown to be incomplete when it was realized that different quantum-Hall states can only be distinguished by more subtle, topological properties ${ }^{1}$. The role of topology as an underlying description of order has since branched out to include topological band insulators and certain featureless gapped Mott insulators with a topological degeneracy in the ground-state wavefunction. Despite intense work, very few candidates for such topologically ordered 'spin liquids' exist. The main difficulty in finding systems that harbour spin-liquid states is the very fact that they violate the Landau paradigm, making conventional order parameters non-existent. Here, we describe a spin-liquid phase in a Bose-Hubbard model on the kagome lattice, and determine its topological order directly by means of a measure known as topological entanglement entropy. We thus identify a non-trivial spin liquid through its entanglement entropy as a gapped ground state with emergent $Z_{2}$ gauge symmetry.

Quantum spin-liquid phases ${ }^{2,3}$ are notoriously elusive, both in real materials and in theoretical models. In part, this is due to the delicate balance of microscopic interaction that must occur so that conventional symmetry-broken order is suppressed at low temperatures. The search is also hampered by the lack of a measurable order parameter, such as magnetization, that would offer a positive indicator of spin-liquid behaviour. Instead, the current procedure of identifying spin liquids involves eliminating all possible order parameters through exhaustive searches of correlation functions ${ }^{4}$. Theoretical work has nonetheless established a classification scheme of gapped spin-liquid states based on the topological degeneracy of their wavefunction ${ }^{2}$. In fact, there exist several model Hamiltonians that have been proposed to contain spin-liquid ground states with the most trivial $Z_{2}$ topological order (corresponding to a four-fold degeneracy on a torus). One of the earliest was the triangular-lattice quantum dimer model ${ }^{5}$ - where dimers are intended to be an effective description of local singlet correlations, not physical spins. Another paradigm in studying topological order has recently emerged in the toric $\operatorname{code}^{6}$, as it is the simplest of a class of exactly solvable models (such as the Levin-Wen models ${ }^{7}$ ) describing different topological quantum field theories. Unfortunately, these models require somewhat artificial multi-spin interaction terms.

In order for a model to be relevant for real physical systems, it is essential to find a spin Hamiltonian with simple two-body interaction terms and a topologically ordered spin-liquid state. One important step in this direction was the introduction of a kagome Bose-Hubbard model ${ }^{8}$. Although this model has a fourspin interaction, it contains a $Z_{2}$ spin liquid over an extended region of its phase diagram. Previously, a related model was shown through quantum Monte Carlo (QMC) studies to have a featureless

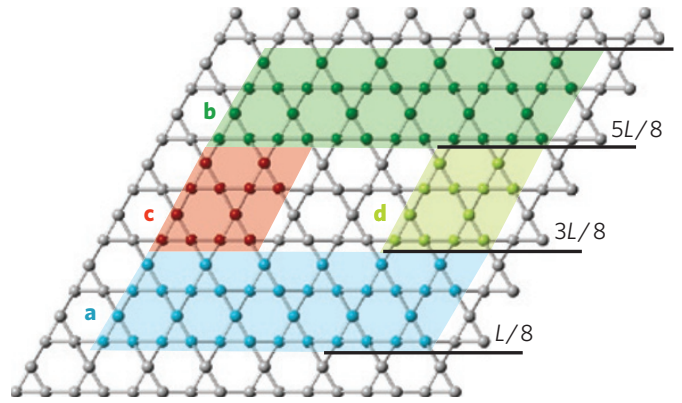

Figure 1 | The kagome lattice used in quantum Monte Carlo simulations. Illustrated is an $L=8$ toroid (a periodic lattice with $8 \times 8 \times 3$ spins). In equation (1), the subregions are $\mathrm{A}_{1}=a \cup b \cup c \cup d, \mathrm{~A}_{2}=a \cup b \cup c$,

$\mathrm{A}_{3}=a \cup b \cup d$, and $\mathrm{A}_{4}=a \cup b$. The width of the annulus is $R=6$, and its thickness is $r=2$.

Mott-insulating state $\mathrm{e}^{9,10}$, where, like most experimental candidates, the absence of order in correlation functions was the main evidence for spin-liquid behaviour.

In 2005 and 2006, several authors ${ }^{11-14}$ identified a quantity $2 \gamma$ called the topological entanglement entropy (EE), which is designed to replace the concept of an 'order parameter' in a topologically ordered system. Based on the idea that the spin-liquid state is a type of collective paramagnet, the topological EE is designed to pick up non-local correlations in the ground-state wavefunction that are not manifest as conventional long-range order. However, these correlations contribute to the total entanglement between different subregions of the system $A$ and its complement $B$ (where $A \cup B$ is the entire system). The EE between A and B can be quantified by the Rényi entropies,

$$
S_{n}(\mathrm{~A})=\frac{1}{1-n} \ln \left[\operatorname{Tr}\left(\rho_{\mathrm{A}}^{n}\right)\right]
$$

where $\rho_{\mathrm{A}}$ is the reduced density matrix of region A. In a topologically ordered state, the non-local entanglement gives a topology-dependent subleading correction to 'area-law' scaling of the EE of subregion A. In 2D, $S_{n}(\mathrm{~A})=a \ell-\gamma j+\cdots$, where $a$ is a non-universal constant, $\ell$ is the boundary length between $\mathrm{A}$ and $\mathrm{B}$, and $j$ is the number of disconnected boundary curves. In Levin and Wen's ${ }^{13}$ construction (used in the calculations in this paper), the topological contribution can be isolated from the area-law scaling (plus any corner contributions) by considering separately the Rényi ${ }^{15}$ entropies on four differently shaped subregions (Fig. 1),

$$
2 \gamma=\lim _{r, R \rightarrow \infty}\left[-S_{n}\left(\mathrm{~A}_{1}\right)+S_{n}\left(\mathrm{~A}_{2}\right)+S_{n}\left(\mathrm{~A}_{3}\right)-S_{n}\left(\mathrm{~A}_{4}\right)\right]
$$

\footnotetext{
${ }^{1}$ Theoretische Physik, ETH Zurich, 8093 Zurich, Switzerland, ${ }^{2}$ Duke University, Department of Physics, Durham, North Carolina, 27708, USA, ${ }^{3}$ Microsoft Research, Station Q, CNSI Building, University of California, Santa Barbara, California, 93106, USA, ${ }^{4}$ Department of Physics and Astronomy, University of Waterloo, Ontario, N2L 3G1, Canada. *e-mail: rgmelko@uwaterloo.ca.
} 

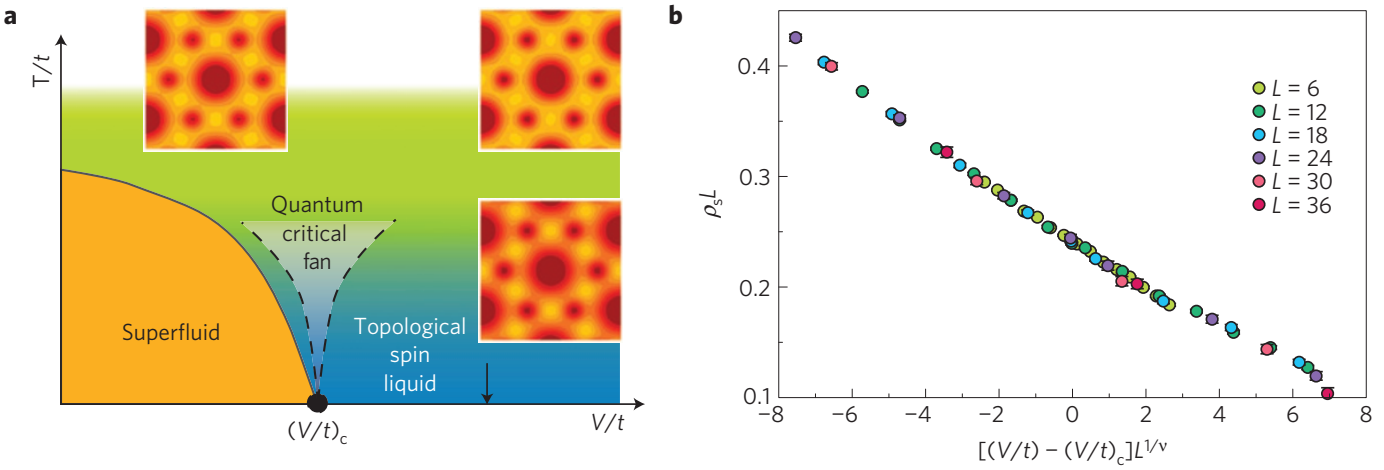

Figure 2 | The phase diagram, structure factors and scaling at the quantum critical point. a, The schematic phase diagram of the kagome Bose-Hubbard model (2). Insets show the structure factor (Fourier transform of the equal-time density-density correlation function) for $V / t=6$ and 8 at high $T$ ( $\beta=3$, top) and low $T(V / t=8$ and $\beta=48$, bottom) in the spin-liquid phase. $\mathbf{b}$, Data collapse of the superfluid density, which in the vicinity of a continuous phase transition should scale as $\rho_{\mathrm{s}}(L, V / t, \beta)=L^{-1} F\left(\left[V / t-(V / t)_{c}\right] L^{1 / v}, \beta / L^{z}\right)$. Here, $F$ is the scaling function, $z$ is the dynamical critical exponent, and $v$ is the correlation-length exponent. It follows from the above equation that if we plot $\rho_{\mathrm{s}} L$ as a function of $\left[V / t-(V / t)_{c}\right] L^{1 / v}$ at fixed $\beta / L^{z}$ then the curves for different system sizes should collapse onto the universal curve $F$ for appropriate values of $v$ and $(V / t)_{c}$, as shown for $v=0.6717,(V / t)_{c}=7.0665$, and $\beta / L=2$.

Naively, because calculating $\gamma$ requires complete knowledge of the ground-state wavefunction (through $\rho_{\mathrm{A}}$ ), previous efforts to calculate it have been restricted to models that can be solved exactly either analytically (for example the toric code) or through numerical exact diagonalization on small size systems (for example the triangular lattice dimer mode $\left.{ }^{16}\right)$. The ability to use $\gamma$ as a general tool to search for and characterize non-trivial topologically ordered phases has been hindered by the inability to access the wavefunction in large-scale numerical methods, namely QMC, currently the only scalable quantum simulation method in 2D and higher. However, with the recent introduction of measurement methods based on the 'replica trick', QMC is now able to access $S_{n}$ (A) for $n \geq 2$ (ref. 17), therefore giving one a method to calculate $\gamma$ in large-scale simulations of quantum spin liquids.

Using stochastic-series-expansion QMC (refs 18,19), we simulate a hard-core Bose-Hubbard model on the kagome lattice, with nearest-neighbour hopping and a six-site potential around each lattice hexagon,

$$
H=-t \sum_{\langle i j\rangle}\left[b_{i}^{\dagger} b_{j}+b_{i} b_{j}^{\dagger}\right]+V \sum_{\bigcirc}\left(n_{\square}\right)^{2}
$$

where $b_{i}^{\dagger}\left(b_{i}\right)$ is the boson creation (annihilation) operator, and $n_{\square}=\sum_{i \in \square}\left(n_{i}-1 / 2\right)$, where $n_{i}=b_{i}^{\dagger} b_{i}$ is the number operator. As mentioned above, variations of this model with more complicated spin interactions are known to harbour a robust spin-liquid ground state $^{8-10}$. In this paper, we consider the simplified Hamiltonian (equation (2)), with only nearest-neighbour hopping, which may be more amenable to construction for example in real cold atomic systems. We observe a transition at low temperature between a superfluid phase and an insulating phase for $(V / t)_{c} \approx 7.0665(15)$ (Fig. 2). For $V / t>(V / t)_{c}$ the superfluid density scales to zero, and density and bond correlators are featureless (similar to the case discussed in ref. 9). This strongly suggests that the insulating phase is a spin liquid. To characterize it, we calculate the topological EE, equation (1) with $n=2$, which for a $Z_{2}$ topological phase should approach $2 \ln (2)$ in the limit $T \rightarrow 0$ (ref. 13). The regions $A_{i}$ are shown in Fig. 1 for an $L=8$ system; these are scaled proportionally for the other system sizes studied in this paper, where $L$ is always a multiple of 8 . Results for $\gamma$ as a function of inverse temperature $\beta=t / T$ are shown in Fig. 3 for several $V / t$.

In the topological phase $(V / t=8)$ we see two distinct plateaux, at differing temperatures, with a non-zero topological $\mathrm{EE}$ as $T \rightarrow 0$. The phenomenon is known to occur in other models, such as the toric $\operatorname{code}^{20}$, where the topological EE at zero temperature of $2 \ln (2)$ can be viewed as a sum of electric and magnetic contributions, each contributing $\ln (2)$. If the electric and magnetic defects have different energies, theory predicts two distinct plateaux corresponding to these individual crossover temperatures ${ }^{20}$, as seen in our data. However, at any fixed non-zero temperature, in the limit of large $L$, the topological $\mathrm{EE}$ vanishes, as the probability of having thermally excited defects in the annulus $A_{1}$ (Fig. 1) tends to unity. Indeed, under the assumption that the probability of having a defect is proportional to $L^{2} \exp \left(-E / k_{\mathrm{B}} T\right)$, where $E$ is the defect energy, the temperature required to see accurate plateaux in the topological EE scales logarithmically with $L$. In Fig. 3, we show finite-size scaling data consistent with this logarithmic scaling.

Our value for the topological EE at the higher- $T$ plateau is indeed very close to $\ln (2)$, becoming more accurately quantized at larger system sizes (Fig. 3b). The value of $2 \ln (2)$ at the lower- $T$ plateau is not as accurately quantized for $L=8$, owing to finite size effects. However, for $Z_{2}$ (and many other) topological theories, the high- $T$ plateau is sufficient to fully characterize the emergent gauge symmetry. Namely, the set of low-energy quasi-particles (either electric or magnetic particles in the $Z_{2}$ case) closes under fusion, and the difference between the upper and lower plateaux equals the logarithm of the total quantum dimension of this set. Therefore, the $Z_{2}$ result can be generalized for other discrete gauge theories, where the high- $T$ plateau is always half of the low- $T$ plateau (note for non-Abelian theories this requires that the low energy particles be electric). Indeed, we observe a high- $T$ plateau consistent with this result, confirming to high accuracy that our spin liquid has an emergent discrete $Z_{2}$ gauge symmetry.

In the superfluid phase, the topological EE tends to zero as $T \rightarrow 0$ (Fig. 3). However, surprisingly, for $V / t=6$ we observe a plateau in the topological EE at intermediate temperatures, $T \sim t$. This behaviour should occur near other transitions out of topological phases. To understand the physics and illustrate the ubiquity of this effect, we consider the same phenomenon in the toric code induced by adding a parallel magnetic field. Consider a square-lattice toriccode Hamiltonian $H=-U \sum_{+} \prod_{i \in+} S_{i}^{z}-g \sum_{\square} \prod_{i \in \square} S_{i}^{x}-h \sum_{i} S_{i}^{z}$, where the first vertex term penalizes vertices that do not have an even number of up spins on the legs of the neighbouring bonds, and the second sum is over plaquettes. Suppose $U \gg g$. By increasing $h / g$, we induce a $T=0$ phase transition from a topological phase to a trivial phase. In a non-zero temperature regime where $U \gg T \gg g$, the problem becomes classical: the quantum dynamics induced by 


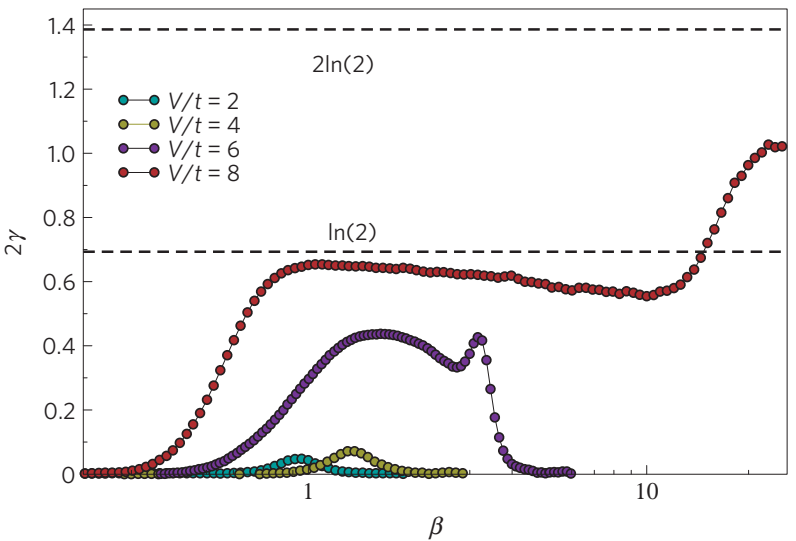

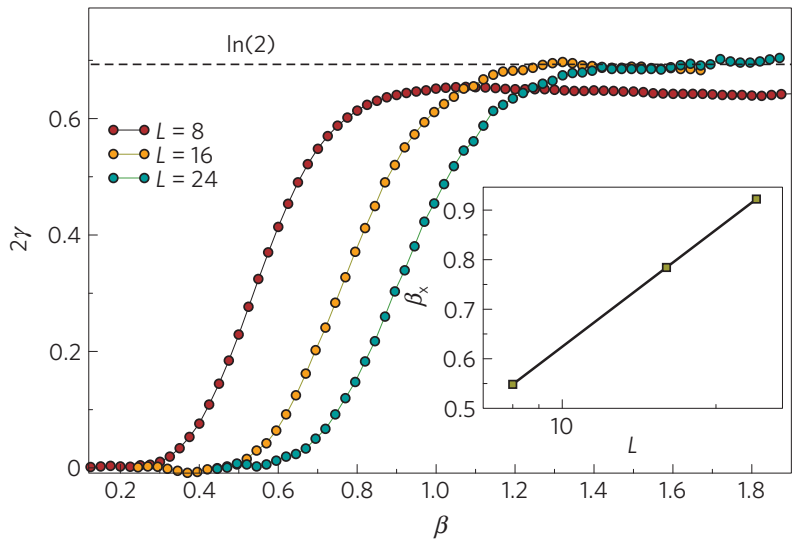

Figure 3 | Quantum Monte Carlo measurement of the topological entanglement entropy. $\mathbf{a}$, The topological entanglement entropy (EE) measured on an $L=8$ system as a function of inverse temperature $\beta=t / T$. The plateaux are a measure of the total quantum dimension ${ }^{13}$, and $\operatorname{should}$ be $\ln (2)$ and $2 \ln (2)$ for a $Z_{2}$ spin liquid. $\mathbf{b}$, The approach of the topological $E E$ to the first plateau, for different system sizes at $V / t=8$. The value of the crossover temperature $\left(\beta_{\mathrm{x}}\right)$, measured at $2 \gamma=\ln (2) / 2$, shows a logarithmic dependence on system size (inset).

$g$ can be ignored and the vertex term restricts us to states described by closed loops of up spins. In this classical problem, there is a phase transition as $h / T$ increases from a topological phase with long loops to a trivial phase with only short loops (this transition is dual to the $2 \mathrm{D}$ Ising transition). Thus, at higher temperatures we see a topological EE, whereas at lower $T$ the topological EE disappears for large enough $\mathrm{h} / \mathrm{g}$. In fact, this kind of physics has been suggested to occur as a 'cooperative paramagnet' in a related kagome lattice model ${ }^{10}$.

The QMC results indicate a quantum critical point, separating the superfluid and topological phases, for a critical $V / t$ located precisely by studying the finite size scaling of the superfluid density $\rho_{\mathrm{s}}$ (Fig. 2 ). The data scales very well with the dynamical exponent $z=1$ and the XY value ${ }^{21}$ for the correlation length exponent $v=0.6717$ at $(V / t)_{c}=7.0665(15)$. This lends support to the prediction that such superfluid-spin liquid critical points may actually of an exotic deconfined type called XY* (refs 22, 23). Measurements of other critical exponents which differ from their XY values, in particular $\eta$, would be required to confirm this prediction. At $T=0$, near the quantum critical point, we expect good quantization of the topological EE whenever $L$ is sufficiently large compared with the correlation length $\xi$, so the topological EE may be controlled by a scaling function of $L / \xi$. At $T>0$, the scaling of the topological EE in the quantum critical fan seems not to have been considered previously; the plateau at intermediate temperatures for $V / t=6$ can perhaps also be understood as a manifestation of increasing $T$ moving one from the zero temperature trivial phase into the quantum-critical fan. Even at $T=0$, the behaviour of constant terms in the entropy at a critical point is largely unexplored, and may depend sensitively on the geometry used to define it. Scaling predicts that near the critical point the topological EE is a function of $\beta / \xi$ and $L / \xi$, implying that in the topological phase, corrections to $2 \ln (2)$ should depend on $(L / \xi)^{2} \exp (-\beta / \xi)$, consistent with a defect energy of order $1 / \xi$. Based on the intermediate temperature plateau at $V / t=6$, it seems likely that only one type of defect, the magnetic defect, becomes gapless at criticality.

To identify a topological phase, it is essential to perform nonlocal probes. Experimentally, such non-local probes could involve braiding operations, as in the proposal of ref. 24, or vison-trapping experiments $^{25,26}$. In this paper, we have shown that the topological entanglement entropy (EE), calculated by QMC using the replica trick, is a practical numerical non-local probe. Other probes might be possible, such as calculating the degeneracy of the ground state on lattices of different topology. However, such a probe suffers from two drawbacks. First, studying surfaces of different Euler characteristic requires the introduction of defects, which is undesirable. Second, although it would be possible in QMC to calculate the degeneracy of the ground state by integrating the specific heat, it requires accurate simulations at a temperature low enough to suppress all excitations - a regime where simulation ergodicity typically becomes a problem. In contrast, we have demonstrated that accurate measurement of topological EE is possible at relatively high temperatures, as long as at least one kind of topologically non-trivial defect is suppressed. Thus, we expect that replica-trick QMC measurements of topological EE will be a fundamental technique in the characterization of non-trivial topological phases in the future.

Received 9 February 2011; accepted 1 June 2011; published online 10 July 2011

\section{References}

1. Wen, X. G. Topological orders in rigid states. Int. J. Mod. Phys. B4, 239-271 (1990).

2. Wen, X. G. Mean-field theory of spin-liquid states with finite energy gap and topological orders. Phys. Rev. B 44, 2664-2672 (1991).

3. Read, N. \& Sachdev, S. Large- $N$ expansion for frustrated quantum antiferromagnets. Phys. Rev. Lett. 66, 1773-1776 (1991).

4. Meng, Z. Y., Lang, T. C., Wessel, S., Assaad, F. F. \& Muramatsu, A. Quantum spin liquid emerging in two-dimensional correlated Dirac fermions. Nature 464, 847-851 (2010).

5. Moessner, R. \& Sondhi, S. L. Resonating valence bond phase in the triangular lattice quantum dimer model. Phys. Rev. Lett. 86, 1881-1884 (2001).

6. Kitaev, A. Fault-tolerant quantum computation by anyons. Ann. Phys. 303, 2-30 (2003).

7. Levin, M. A. \& Wen, X. G. String-net condensation: A physical mechanism for topological phases. Phys. Rev. B 71, 045110 (2005).

8. Balents, L., Fisher, M. P. A. \& Girvin, S. M. Fractionalization in an easy-axis kagome antiferromagnet. Phys. Rev. B 65, 224412 (2002).

9. Isakov, S. V., Kim, Y. B. \& Paramekanti, A. Spin-liquid phase in a spin-1/2 quantum magnet on the kagome lattice. Phys. Rev. Lett. 97, 207204 (2006).

10. Isakov, S. V., Paramekanti, A. \& Kim, Y. B. Exotic phase diagram of a cluster charging model of bosons on the kagome lattice. Phys. Rev. B 76, 224431 (2007).

11. Hamma, A., Ionicioiu, R. \& Zanardi, P. Ground state entanglement and geometric entropy in the Kitaev model. Phys. Lett. A 337, 22-28 (2005).

12. Hamma, A., Ionicioiu, R. \& Zanardi, P. Bipartite entanglement and entropic boundary law in lattice spin systems. Phys. Rev. A 71, 022315 (2005).

13. Levin, M. \& Wen, X. G. Detecting topological order in a ground state wave function. Phys. Rev. Lett 96, 110405 (2006).

14. Kitaev, A. \& Preskill, J. Topological entanglement entropy. Phys. Rev. Lett. 96, 110404 (2006).

15. Flammia, S. T., Hamma, A., Hughes, T. L. \& Wen, X. G. Topological entanglement Rényi entropy and reduced density matrix structure. Phys. Rev. Lett. 103, 261601 (2009). 
16. Furukawa, S. \& Misguich, G. Topological entanglement entropy in the quantum dimer model on the triangular lattice. Phys. Rev. B 75, 214407 (2007).

17. Melko, R. G., Kallin, A. B. \& Hastings, M. B. Finite-size scaling of mutual information in Monte Carlo simulations: Application to the spin- $\frac{1}{2} x x z$ model. Phys. Rev. B 82, 100409 (2010).

18. Sandvik, A. W. \& Kurkijärvi, J. Quantum Monte Carlo simulation method for spin systems. Phys. Rev. B 43, 5950-5961 (1991).

19. Sandvik, A. W. A generalization of Handscomb's quantum Monte Carlo scheme-application to the 1-d Hubbard model. J. Phys. A 25, 3667-3682 (1992).

20. Castelnovo, C. \& Chamon, C. Entanglement and topological entropy of the toric code at finite temperature. Phys. Rev. B 76, 184442 (2007).

21. Campostrini, M., Hasenbusch, M., Pelissetto, A. \& Vicari, E. Theoretical estimates of the critical exponents of the superfluid transition in ${ }^{4} \mathrm{He}$ by lattice methods. Phys. Rev. B 74, 144506 (2006).

22. Chubukov, A. V., Senthil, T. \& Sachdev, S. Universal magnetic properties of frustrated quantum antiferromagnets in two dimensions. Phys. Rev. Lett. 72, 2089-2092 (1994).

23. Chubukov, A. V., Sachdev, S. \& Senthil, T. Quantum phase transitions in frustrated quantum antiferromagnets. Nucl. Phys. B 426, 601-643 (1994).

24. Das Sarma, S., Freedman, M. \& Nayak, C. Topologically protected qubits from a possible non-Abelian fractional quantum Hall state. Phys. Rev. Lett. 94, 166802 (2005).
25. Senthil, T. \& Fisher, M. P. A. Fractionalization in the cuprates: Detecting the topological order. Phys. Rev. Lett. 86, 292-295 (2001).

26. Sedgewick, R. D., Scalapino, D. J. \& Sugar, R. L. Fractionalized phase in an $x y-z_{2}$ gauge model. Phys. Rev. B 65, 054508 (2002).

\section{Acknowledgements}

The authors thank M. A. Levin for explaining the high-temperature plateau in discrete gauge theories and $\mathrm{T}$. Senthil for elucidating the $\mathrm{XY}^{*}$ nature of the quantum critical point. This work has been supported by the Natural Sciences and Engineering Research Council of Canada (NSERC), NSF grant No PHY 05-51164 (KITP) and the Swiss $\mathrm{HP}^{2} \mathrm{C}$ initiative. Simulations were performed on the Brutus cluster at ETH Zurich and the computing facilities of SHARCNET.

\section{Author contributions}

S.V.I. developed the quantum Monte Carlo code and carried out the simulations. All three authors analysed the results and wrote the paper.

\section{Additional information}

The authors declare no competing financial interests. Reprints and permissions information is available online at http://www.nature.com/reprints. Correspondence and requests for materials should be addressed to R.G.M. 